\title{
Dynamic barriers and tunneling. New views of hydrogen transfer in enzyme reactions*
}

\author{
Judith P. Klinman \\ Department of Chemistry and Department of Molecular and Cell Biology, \\ University of California, Berkeley, CA 94720, USA
}

\begin{abstract}
Hydrogen-transfer processes are expected to show appreciable quantum mechanical behavior. Intensive investigations of enzymes under their physiological conditions show this to be true in practically every example investigated. Initially, tunneling was treated either as a tunneling correction [cf. Bell, The Tunnel Effect in Chemistry, Chapman \& Hall, New York, (1980)], or as corner-cutting [Truhlar et al., J. Chem. Phys. 100, 12771 (1996)]. This worked well as long as the observed properties could be explained by "corrections" to transition-state theory. However, over the past several years, enzymatic behaviors have been observed that are so deviant as to lie outside of transition-state theory. This phenomenon is discussed in the context of the enzyme, soybean lipoxygenase. An environmentally coupled hydrogen-tunneling model is presented that derives from the treatments of Kuznetsov and Ullstrup [Can. J. Chem. 77, 689 (1999)], and includes heavy-atom reorganization (temperature-dependent and largely isotope-independent), together with heavy-atom gating (temperature- and isotope-dependent). This treatment can explain a wide range of behaviors and leads to a new view of the origin of kinetic isotope effects in hydrogen-transfer reactions. These properties link enzyme fluctuations to the hydrogen-transfer reaction coordinate, making a quantum view of $\mathrm{H}$-transfer necessarily a dynamic view of catalysis.
\end{abstract}

\section{INTRODUCTION}

The discipline of enzyme catalysis has been "anchored" by transition-state (TS) theory for almost half a century. Starting with the hypothesis of Pauling [1], the rate enhancement of enzymes has been attributed to a greater affinity of enzymes for their substrates in the TS, than in the ground state. This approach has offered a relatively simple context in which to compare enzymatic and nonenzymatic rate phenomena and has stimulated many exciting areas of research that include the design of TS analogs [2] as potential drugs and the entire field of catalytic antibodies [3]. However, the concept of enhanced TS binding is simply a restatement of catalysis in the context of TS theory, and may not have the physical reality of tighter TS binding that has been traditionally inferred.

In the context of enhanced TS binding for enzyme catalysis, a primary focus has been on the atoms at the reaction center that are being broken or made and the attendant reduction in the barrier height for this process. In more sophisticated pictures, the entire barrier shape, including barrier width, would be included, as would the contribution of motion within the protein itself. This manuscript concerns itself with recent studies that force us to move beyond simple TS theory in our conceptualization of enzyme-catalyzed hydrogen-transfer reactions. These observations are likely to have general impli-

\footnotetext{
*Plenary lecture presented at the $16^{\text {th }}$ International Conference on Physical Organic Chemistry (ICPOC-16): Structure and Mechanism in Organic Chemistry, San Diego, California, USA, 4-9 August 2002. Other presentations are published in this issue, pp. 541-630.

ॠCorresponding author: E-mail: klinman@socrates.berkeley.ed
} 
cations for solution reactions of hydrogen and, importantly, for the interpretation of physical organic probes that are traditionally used to infer TS structure.

\section{ROLE OF HYDROGEN TUNNELING IN ENZYMES}

It is well accepted that electrons are transferred from donor to acceptor by quantum mechanical tunneling and that the observed rates and energies of activation derive from heavy-atom motions. Marcus theory provides an elegant description of this behavior, showing how the activation energy for the process, $\Delta G^{\ddagger}$, is determined by the parameters $\lambda$, an environmental distortion that must precede the electron transfer, and $\Delta G^{\circ}$, the reaction driving force [4]. Though much larger than the electron, the de Broglie wavelength for the proton is estimated to be in the same range as the distance over which hydrogen will typically be transferred from donor to acceptor atom (ca. 0.6-0.7 $\AA$ ) [5]. This has led to the expectation of considerable quantum mechanical behavior for hydrogen transfer, though hydrogen transfer reactions often "appear to behave classically".

A useful early approach that incorporated the quantum mechanical property of hydrogen was introduced by Bell [6] and is illustrated in Fig. 1.

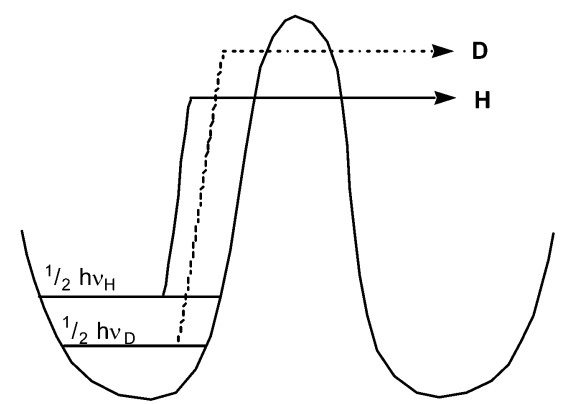

Fig. 1 Illustration of a classical reaction coordinate for H-transfer with tunneling of $\mathrm{H}$ and $\mathrm{D}$ below the top of the barrier.

This shows a hydrogen-transfer coordinate where both the light and heavy particles (H and D) have sufficient wave property that they can move through the barrier. This is likely to occur near the top of the barrier, where it is more narrow, and to occur at a lower point, i.e., with more tunneling, for the lighter particle $(\mathrm{H})$. The type of tunnel correction that is shown in Fig. 1 makes a number of predictions regarding deviations from classical behavior. These concern (i) the relative rates for the three isotopes of hydrogen $(\mathrm{H}, \mathrm{D}$, and $\mathrm{T})$ and (ii) their temperature dependencies.

With regard to the former, referred to as the Swain-Schaad relationship, a simple mass-rate relationship is expected to break down when tunneling occurs, due to the greater facility of tunneling with the lighter isotopes. This has been shown to be most manifest when the heaviest isotope, tritium, is used as a frame of reference [cf. 7,8]:

$$
\begin{array}{ll}
\text { Classical: } & \left(k_{\mathrm{D}} / k_{\mathrm{T}}\right)^{3.3}=\left(k_{\mathrm{H}} / k_{\mathrm{T}}\right) \\
\text { Tunnel correction: } & \left(k_{\mathrm{D}} / k_{\mathrm{T}}\right)^{3.3}<\left(k_{\mathrm{H}} / k_{\mathrm{T}}\right)
\end{array}
$$

According to eq. 1, a well-defined relationship among the three isotopes can be expected when rate differences arise from zero point energy differences. The exponent can actually exceed 3.3 under special instances without tunneling, but will not get larger than ca. 4.5 [9]. By contrast, when tunneling begins to dominate, eq. 2, the exponent relating $\mathrm{D} / \mathrm{T}$ and $\mathrm{H} / \mathrm{T}$ isotope effects can become greatly inflated, owing to the greater ease of tunneling for $\mathrm{H}$ than $\mathrm{D}[7,8]$. 
The second expectation from the Bell correction is an onset of curvature in Arrhenius plots as temperature is reduced, arising from an increase in rate due to tunneling [6]. Using observed data from an experimentally accessible temperature range to extrapolate to infinite temperature, the greater tunneling particle (e.g., $\mathrm{H}$ in relation to $\mathrm{D}$ ) incorporates greater curvature into its temperature dependence. This produces a crossing of isotopic lines, such that isotope effects on apparent Arrhenius prefactors fall below unity:

Classical:

$$
\mathrm{A}_{\mathrm{H}} / \mathrm{A}_{\mathrm{D}} \cong \mathrm{A}_{\mathrm{H}} / \mathrm{A}_{\mathrm{T}} \cong \mathrm{A}_{\mathrm{D}} / \mathrm{A}_{\mathrm{T}} \cong 1
$$

Tunnel correction: $\quad A_{H} / A_{D}, A_{H} / A_{T}, A_{D} / A_{T}<1$

In practice, enzyme-catalyzed reactions present excellent systems for detecting tunneling. This is because of their high product specificity and stereochemistry, allowing a straightforward analysis of substrate disappearance/product appearance and a clear distinction between primary and secondary isotope effects. There are now a number of examples of enzymes that show highly deviant Swain-Schaad relationships for secondary isotope effects in hydride-transfer reactions $[5,10,11]$ and several different classes of reactions that demonstrate $\mathrm{A}_{1} / \mathrm{A}_{2}<<1[12-16]$. The latter span reaction mechanisms that involve hydride, proton, and hydrogen atoms transfer, indicating that the role of tunneling is unrelated to the species that is being transferred.

\section{“BREAKING” THE TUNNEL CORRECTION}

The Bell correction suffers from the limitations inherent in TS theory, because it is essentially a correction to a semiclassical treatment of $\mathrm{H}$-transfer reactions. In this respect, it focuses exclusively on the hydrogen reaction coordinate and ignores contributions from the surrounding heavy-atom environment. By contrast, $\mathrm{H}$-tunneling is expected to be exquisitely sensitive to the relative energies of the reactant and product and their distances, making the tunneling phenomenon a multidimensional process. The Bell description does allow for a full tunneling picture at very low temperatures, where neither the proton nor deuteron has sufficient thermal energy to move over the barrier. In this low-temperature regime [6], all isotopes react by tunneling, giving rise to the expectation of enormous isotope effects, isotope effects on the Arrhenius prefactors that equal the isotope effect and the absence of an energy of activation.

In the last four years, a growing number of enzyme systems have been demonstrated to have properties that simply cannot be fit by the Bell correction [11,17-22]. These are summarized in Table 1,

Table 1 Enzyme reactions with properties outside the "tunnel correction".

\begin{tabular}{lccc}
\hline Enzyme & $k_{\mathrm{H}} / k_{\mathrm{D}}$ & $\mathrm{A}_{\mathrm{H}} / \mathrm{A}_{\mathrm{D}}$ & Ref. \\
\hline $\mathrm{SLO}^{\mathrm{a}}$ & 81 & 18 & 16 \\
$\mathrm{ht}^{\mathrm{ADDH}} \mathrm{b}^{\mathrm{b}}$ & 3.2 & 2.2 & 10 \\
$\mathrm{PHM}^{\mathrm{c}}$ & 10 & 5.9 & 17 \\
$\mathrm{MADH}^{\mathrm{d}}$ & 17 & 13 & 18 \\
TMADH $^{\mathrm{e}}$ & 4.6 & 7.8 & 19 \\
SADH $^{\mathrm{f}}$ & 7.3 & 5.8 & 20 \\
AcCoA desat. $^{\mathrm{g}}$ & 23 & 2.2 & 21 \\
\hline
\end{tabular}

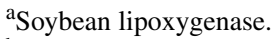

${ }^{\mathrm{b}}$ High-temperature alcohol dehydrogenase.

${ }^{c}$ Peptidylglycine $\alpha$-hydroxylating monooxygenase.

${ }^{\mathrm{d}}$ Methylamine dehydrogenase.

e Trimethylamine dehydrogenase.

${ }^{\mathrm{f}}$ Sarcosine dehydrogenase.

${ }^{\mathrm{g}}$ AcylCoA desaturase. 
where in most systems, significant effort has been expended to demonstrate that H-transfer is rate-limiting in the experimental temperature range. In several instances, there is a pattern of greatly elevated isotope effects and in every case, the ratio of $A_{1} / A_{2}$ is larger than unity. Though not indicated in Table 1, each example also has a nonzero enthalpy of activation for the H-transfer process. It is impossible to attribute these phenomena to an isolated abnormality, with the weight of the data necessitating a new formalism for $\mathrm{H}$-transfer reactions.

\section{SOYBEAN LIPOXYGENASE REACTION}

We have used the soybean lipoxygenase (SLO) reaction as a paradigmatic system in which to explore an alternate view of $\mathrm{H}$-transfer in condensed phase. The SLO reaction is known to occur within a buried active site and to involve a net hydrogen atom transfer from an internal position of a bound fatty acid $\left[\mathrm{C} 11\right.$ of linoleic acid (LA)] to an active site $\mathrm{Fe}^{3+}-\mathrm{OH}$ (Scheme 1).

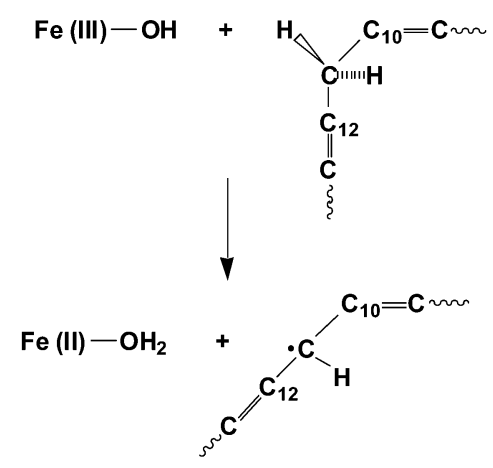

Scheme 1 Postulated mechanism for hydrogen atom transfer in SLO.

The products of this reaction, a substrate-derived free radical and $\mathrm{Fe}^{2+}-\mathrm{OH}_{2}$, subsequently react with $\mathrm{O}_{2}$ to complete the reaction cycle [23]. Using chirally labeled substrates, the origin of the elevated isotope effect has been shown to be due to the primary $(11, \mathrm{~S}-\mathrm{H})$ position, with a small, normal secondary $(11, \mathrm{R}-\mathrm{H})$ effect of 1.1 [24]. The temperature dependencies of the $\mathrm{C}-\mathrm{H}$ activation for $\mathrm{H}-$ and D-LA are linear between 5 and $50{ }^{\circ} \mathrm{C}$ and show the following Eyring parameters and their isotope effects [17]:

Parameters: $\Delta H^{\ddagger}=1.5 \mathrm{kcal} / \mathrm{mol} ;-T \Delta S^{\ddagger}=12.8 \mathrm{kcal} / \mathrm{mol}$

Isotope effects on parameters: $\Delta \Delta H^{\ddagger}=0.9 \mathrm{kcal} / \mathrm{mol} ;-T \Delta \Delta S^{\ddagger}=1.8 \mathrm{kcal} / \mathrm{mol}$

These indicate that the major barrier to reaction and the dominant isotope effect are in $-T \Delta S^{\ddagger}$ and $-T \Delta \Delta S^{\ddagger}$, respectively. How can this be?

Over the last decade, a number of authors have presented theoretical approaches to H-transfer that treat the $\mathrm{H}$-coordinate as fully quantum mechanical and incorporate various degrees of heavy-atom motion to describe the observed thermal barriers to reaction [25-29]. We have used a modification of a treatment $[29,17]$ that incorporates this multidimensionality of the H-transfer process and focuses attention on the role of the surrounding heavy-atoms. The most straightforward analysis is shown in eq. 5, where the preexponential constant contains the electronic coupling term and the first exponential describes the well-known Marcus term from electron-transfer theory [4]. The second exponential is the Frank-Condon term for the wave function overlap and is described in terms of the mass $\left(\mathrm{m}_{\mathrm{H}}\right)$ and frequency $\left(\omega_{\mathrm{H}}\right)$ of the transferred particle as well as the distance of transfer $\left(\mathrm{r}_{\mathrm{H}}\right)$. In eq. $5, \hbar$ is Planck's constant divided by $2 \pi, \mathrm{R}$ is the gas constant, and $\mathrm{T}$ is temperature.

The enthalpic barrier to reaction comes from the Marcus term that reflects the fact that tunneling only occurs from isoenergetic states; thus, unless $K_{\mathrm{eq}}=1$ for H-transfer, energy must be put into the system to achieve "transient degeneracy", giving rise to the characteristic temperature dependence. The 


$$
k_{\text {tun }}=(\text { Const. }) \exp \left\{-\left(\Delta G^{\circ}+\lambda\right)^{2} /{ }_{(4 \lambda R T)}\right\} \exp ^{\left(-m_{H} \omega_{H} r_{H}^{2} / 2 \hbar\right)}
$$

isotope dependence derives from the Frank-Condon term and is most critically dependent on the distance of transfer. As originally pointed out by Kuznetsov and Ullstrup [29], the data for wild-type SLO (WT-SLO) can be fitted by the expression in eq. 5 .

The story, however, becomes a great deal more informative when we analyze mutants of SLO. The residues that were chosen for site-specific mutagenesis are hydrophobic residues in the vicinity of the bound substrate and iron center. We chose to focus on Leu 754 and Leu 546, which sandwich the reactive $\mathrm{C} 11$ position of substrate, and Ile 553, which is one helix turn away. In each instance, the active site residue was converted to alanine and studied as a function of substrate deuteration and temperature. The results are summarized in Table 2 .

Table 2 Experimental parameters for WT-SLO and mutants [17] $]^{\mathrm{a}}$.

\begin{tabular}{lccccc}
\hline & $\begin{array}{c}k_{\mathrm{cat}} \\
\left(\mathrm{s}^{-1}\right)\end{array}$ & $\begin{array}{c}E_{\mathrm{act}} \\
(\mathrm{kcal} / \mathrm{mol})\end{array}$ & $\begin{array}{c}\Delta E_{\mathrm{act}} \\
(\mathrm{kcal} / \mathrm{mol})\end{array}$ & $\begin{array}{c}k_{\mathrm{H}} / k_{\mathrm{D}} \\
\left(30{ }^{\circ} \mathrm{C}\right)\end{array}$ & $\mathrm{A}_{\mathrm{H}} / \mathrm{A}_{\mathrm{D}}$ \\
\hline $\mathrm{WT}$ & $260(12)$ & $2.2(0.2)$ & $0.8(0.2)$ & $84(8)$ & $19(6)$ \\
$\mathrm{Leu}^{546} \rightarrow$ Ala & $48(0.6)$ & $4.1(0.4)$ & $1.9(0.6)$ & $93(9)$ & $4(4)$ \\
$\mathrm{Leu}^{754} \rightarrow$ Ala & $0.31(0.021)$ & $4.1(0.3)$ & $2.0(0.5)$ & $112(11)$ & $3(3)$ \\
$\mathrm{Ile}^{553} \rightarrow$ Ala & $280(10)$ & $1.9(0.2)$ & $4.0(0.3)$ & $93(4)$ & $0.12(0.06)$ \\
\hline
\end{tabular}

${ }^{\mathrm{a}}$ Experimental error is indicated in parenthesis.

One aspect that needed to be established was whether the observed changes in $k_{\text {cat }}$ and $E_{\text {act }}$ (Table 2) were due to effects of the protein mutation on substrate binding. For this purpose, we compared the changes in free energy for each mutant enzyme relative to WT-SLO under conditions of substrate saturation $\left(\Delta \Delta G^{\ddagger}\right.$ for the $k_{\text {cat }}$ parameter) to conditions of low substrate where the rate contains substrate binding as well as catalysis ( $\Delta \Delta G^{\ddagger}$ for the $k_{\text {cat }} / K_{\mathrm{m}}$ parameter). Importantly, the trends in $\Delta \Delta G^{\ddagger}$ are essentially identical under both conditions, indicating that the same effects dominate $k_{\text {cat }}$ and $k_{\text {cat }} / K_{\mathrm{m}}$. This rules out significant contributions from changes in substrate binding among the mutant enzymes [17].

The key observations of Table 2 are shown in columns 4 and 5, which indicate isotope effects near 100 for WT-SLO and the mutants, together with isotope effects on the Arrhenius prefactors that decrease from 19-0.12. Implicit in eq. 5 is a small dependence of $A_{H} / A_{D}$ on the reaction driving force. This comes from the fact that some tunneling will take place from excited vibrational states of the substrate $\mathrm{C}-\mathrm{H}$ bond, with excited vibrational modes playing a more significant role for the $\mathrm{D}$ - than $\mathrm{H}$-substrate. However, when data are simulated by varying $\Delta G^{\circ}$ between 0 to $-30 \mathrm{kcal} / \mathrm{mol}, \mathrm{A}_{\mathrm{H}} / \mathrm{A}_{\mathrm{D}}$ is found to decrease only modestly and to plateau significantly above 10 . This indicates that the simple physical picture represented by eq. 5 is not sufficient to explain the full set of data for SLO.

The final model that has been invoked is shown in eq. 6 , and includes a gating term that is the integral of the product of the Frank-Condon term and an exponential term that contains the probability of the protein achieving a range of transfer distances between the hydrogen donor and acceptor [17].

$$
k_{\text {tun }}=(\text { Const. }) \exp \left\{-\left(\Delta G^{\circ}+\lambda\right)^{2} /{ }_{(4 \lambda R T)}\right\} \int_{r_{1}}^{r_{0}} \exp ^{\left\{-m_{H} \omega_{H} r_{H}^{2} / 2 \hbar\right\}} \exp ^{\left\{-E_{X} / k_{b} T\right\}} d X
$$

According to eq. $6, E_{x}=\hbar \omega_{\mathrm{x}} \mathrm{X}^{2}$, where $\omega_{\mathrm{x}}$ is the frequency of the protein gating mode. The term $\mathrm{X}=r_{x} \sqrt{m_{x} \omega_{x} / \hbar}$ where $m_{x}$ and $r_{x}$ are the mass of the gating unit and the distance the gating unit moves, respectively. This integral predicts an optimal distance for $\mathrm{H}$ and $\mathrm{D}$ tunneling, with this distance being shorter for the heavier isotope. This integral in eq. 6 is the principal origin of the temperature dependence of the isotope effect and produces a range of values for $A_{H} / A_{D}$ that depend on the magnitude of $\omega_{x}$. 
The fitting of the complete set of data for WT-SLO and its mutants is summarized in Table 3 . This shows (right side of Table 3 ) that 15 out of 16 experimental parameters are well fitted.

Table 3 Input and calculated parameters for SLO [17] using eq. 6 in text.

\begin{tabular}{lcccc|cccc}
\hline & $\begin{array}{c}-\Delta G^{\mathrm{o}} \\
(\mathrm{kcal} / \mathrm{mol})\end{array}$ & $\begin{array}{c}\mathrm{r}_{\mathrm{o}} \\
(\AA)\end{array}$ & $\begin{array}{c}\omega_{\mathrm{x}} \\
\left(\mathrm{cm}^{-1}\right)\end{array}$ & $\begin{array}{c}\mathrm{E}_{\mathrm{a}} \\
(\mathrm{kcal} / \mathrm{mol})\end{array}$ & $\Delta E_{\mathrm{act}}$ & $\mathrm{A}_{\mathrm{H}} / \mathrm{A}_{\mathrm{D}}$ & $\begin{array}{c}\mathrm{KIE} \\
\left(30^{\circ}\right)\end{array}$ \\
\hline $\mathrm{WT}$ & 6 & 19.5 & 0.6 & 400 & 2.8 & 1.2 & 12.4 & 93 \\
$\mathrm{Leu}^{546} \rightarrow \mathrm{Ala}$ & 6 & 19.5 & 0.7 & 165 & 4.0 & 1.9 & 4.1 & 91 \\
$\mathrm{Leu}^{754} \rightarrow \mathrm{Ala}$ & 6 & 19.5 & 0.7 & 165 & 4.0 & 1.9 & 4.1 & 91 \\
$\mathrm{Ile}^{553} \rightarrow \mathrm{Ala}^{\mathrm{a}}$ & 6 & 19.5 & 1.0 & 89 & $(8.1)$ & 3.0 & 0.57 & 95 \\
\hline
\end{tabular}

${ }^{\mathrm{a}} \mathrm{r}_{1}=0.6 \AA(\mathrm{H})$ and $0.53 \AA(\mathrm{D})$ at $30^{\circ} \mathrm{C}$.

Only a single parameter is not matched at all, $E_{\text {act }}$ for Ile553 $\rightarrow$ Ala. This is almost certainly due to the fact that both $\Delta G^{\mathrm{o}}$ and $\lambda$ have been held constant for all enzyme forms, and implies that $\lambda$ may be reduced for Ile553 $\rightarrow$ Ala in relation to WT-SLO and the other mutants. Work is currently in progress to examine the impact of a series of amino acid side chain substitutions at position 553 on the experimentally observed rate and isotope effect parameters.

The extensive data for WT-SLO and its mutants show that the experimental trend in $A_{H} / A_{D}$ is mirrored by a decrease in $\omega_{\mathrm{x}}$. The absolute values for $\omega_{\mathrm{x}}$, which were obtained by assuming a mass for the gating motion of the protein of $110 \mathrm{Da}$, are less important than their trend. The latter indicates a progressive "softening" of the potential for protein motion as the bulk of residues that line the substrate site is decreased. For WT-SLO, the high value of $\omega_{\mathrm{x}}$, which leads to $\hbar \omega_{\mathrm{x}}$ greater than $k \mathrm{~T}$ (where $k$ is the Boltzmann constant and $\mathrm{T}$ is temperature), implies a fairly stiff active site that is well optimized for $\mathrm{H}$-tunneling and requires relatively little gating. By contrast, the space created by replacement of the bulky side chains at positions 754,546 , and 553 with alanine produces a softening of the protein potential and a need for increased gating to achieve active site configurations suitable for tunneling. This decrease in $\omega_{\mathrm{x}}$ with the mutants will produce values for $\hbar \omega_{\mathrm{x}}$ that are comparable or less than $k \mathrm{~T}$, leading to the expectation of significant activation of the protein-gating modes under the condition of ambient temperature.

With protein systems other than SLO, the degree of gating is expected to depend on the specific features of the active site, in particular how well the site is optimized for H-tunneling. In recent studies of the peptidylglycine $\alpha$-hydroxylating monooxygenase (PHM, Table 1), a copper enzyme with a highly solvent exposed active site, the size of the observed isotope effects and their temperature dependencies have led to a calculated value for $\omega_{\mathrm{x}}$ that is only $45 \mathrm{~cm}^{-1}$, implying considerably more gating than for WT-SLO [18]. Interestingly, preliminary analysis of a mutant SLO in which the bulk at Ile553 has been further reduced by insertion of glycine [30], shows a value for $\omega_{\mathrm{x}}\left(47 \mathrm{~cm}^{-1}\right)$ that is almost identical to that seen with the PHM enzyme.

\section{IMPLICATIONS FOR THEORIES OF H-TRANSFER IN CONDENSED PHASE}

A key aspect of the models given in eqs. 5 and 6 is that all of the temperature dependence of the reaction rate comes from the heavy-atom motions, i.e., $E_{\text {act }}$ is unrelated to the hydrogen-transfer coordinate itself. We have been increasingly concerned with the nature of the protein motions that constitute these heavy-atom terms, finding it useful to divide the motions into two categories: passive and active. In this scheme, the passive motions refer to the first exponential in eq. 6 that describes the sampling of configurations that differ with regard to the relative energy levels of reactant and product. This reflects a Boltzmann distribution of states that are in equilibrium with the environment, with only some (a relatively few) configurations being optimal for catalysis. The protein can be thought of as a "jiggling bowl 
of jello" that allows changes in dipolar interactions that alter $K_{\text {eq }}$ values for the interconversion of the reactant and product states. The active dynamics are contained within the integral of eq. 6 and reflect the motions that change the reaction barrier width. This is fundamentally a different kind of motion, since it represents a vectorial coupling of protein motion to the reaction coordinate. This latter motion is less easily understood, because it implies relatively large energy changes, to allow heavy-atoms to approach closer than their van der Waals distances, as well as an implicit directionality. It is this "active" motion that presents the greatest challenge to our understanding of the link between protein dynamics and catalysis.

The introduction of a new conceptual framework for H-transfer in condensed phase has fundamental implications for the application of physical organic probes, such as structure-activity correlations and isotope effects. For example, in contrast to the Westheimer model [31] that relates the size of primary isotope effects to TS structure, the work described herein relates the size and temperature dependence of the primary isotope effect to the distance between the donor and acceptor atoms and the gating motions that impact this distance. As shown in Fig. 2, an entire range of behaviors is possible in which there is little gating $\left(\hbar \omega_{\mathrm{x}}>k \mathrm{~T}\right)$, such that the isotope effect appears large and close to temperature independent, to a large degree of gating $\left(\hbar \omega_{\mathrm{x}}<k \mathrm{~T}\right)$, where the isotope effects can become fairly small and the temperature dependence of the isotope effect is very pronounced. We are at an exciting juncture in our understanding of the fundamental and biologically important $\mathrm{H}$-transfer reaction, as TS theory is giving way to a dynamical, multidimensional description of the reaction coordinate.

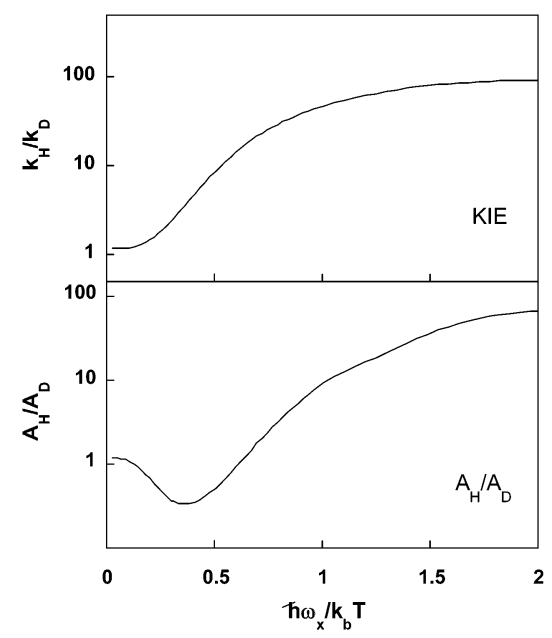

Fig. 2 Environmentally gated tunnel model (cf., eq. 6 in text) in which the temperature dependence and size of isotope effects varies as a function of $\omega_{\mathrm{x}}$.

\section{ACKNOWLEDGMENTS}

Many of the ideas in this manuscript were developed with Dr. Mike Knapp, currently at the Department of Chemistry, University of Massachusetts, Amherst and supported by grants from the NIH (GM25765) and the NSF (MCB 0135446). 


\section{REFERENCES}

1. L. Pauling. Amer. Scientist 36, 51 (1948).

2. R. Wolfenden. Nature 223, 704 (1969).

3. P. G. Schult and R. A. Lerner. Acc. Chem. Res. 26, 391 (1993).

4. R. A. Marcus and N. Sutin. Biochem. Biophys. Acta 811, 265 (1985).

5. Y. Cha, C. J. Murray, J. P. Klinman. Science 243, 1325 (1989).

6. R. P. Bell. The Tunnel Effect in Chemistry, Chapman \& Hall, New York (1980).

7. W H. Saunders. J. Am. Chem. Soc. 107, 164 (1985).

8. K. L. Grant and J. P. Klinman. Bioorg. Chem. 20, 1 (1992).

9. A. Kohen and J. H. Jensen. J. Am. Chem. Soc. 124, 3858 (2002).

10. B. J. Bahnson, T. D. Colby, J. K. Chin, B. M. Goldstein, J. P. Klinman. Proc. Natl. Acad. Sci. USA 94, 12797 (1997).

11. A. Kohen, R. Cannio, S. Bartolucci, J. P. Klinman. Nature 399, 496 (1999).

12. K. Grant and J. P. Klinman. Biochemistry 28, 6597 (1989).

13. T. Jonsson, D. E. Edmondson, J. P. Klinman. Biochemistry 33, 14871 (1994).

14. S. L. Seymour and J. P. Klinman. Biochemistry 41, 8747 (2002).

15. M. M. Whittaker, D. P. Ballou, J. W. Whittaker. Biochemistry 37, 8426 (1998).

16. S. Chowdbury and R. Banerjee. J. Am. Chem. Soc. 122, 5417 (2000).

17. M. J. Knapp, K. Rickert, J. P. Klinman. J. Am. Chem. Soc. 124, 8194 (2002).

18. W. A. Francisco, M. J. Knapp, N. J. Blackburn, J. P. Klinman. J. Am. Chem. Soc. 124, 8194 (2002).

19. J. Basran, M. J. Sutcliffe, N. S. Scrutton. Biochemistry 38, 3218 (1999).

20. J. Basran, M. J. Sutcliffe, N. S. Scrutton. J. Biol. Chem. 276, 24581 (2001).

21. R. J. Harris, R. Meskys, N. J. Sutcliffe, N. S. Scrutton. Biochemistry 39, 1189 (2000).

22. J. L. Abad, F. Camps, G. Fabrias. Angew. Chem., Int. Ed. 39, 3279 (2000).

23. M. H. Glickman and J. P. Klinman. Biochemistry 35, 12882 (1996).

24. K. W. Rickert and J. P. Klinman. Biochemistry 38, 12218 (1999).

25. D. Borgis and J. T. Hynes. J. Phys. Chem. 100, 1118 (1996).

26. W. J. Bruno and W. Bialek. Biophys. J. 63, 689 (1992).

27. D. Antoniou and S. D. Schwartz. Proc. Natl. Acad. Sci. USA 94, 12360 (1997).

28. S. Hammes-Schiffer. Acc. Chem. Res. 34, 273 (2001).

29. A.M. Kuznetsov and J. Ulstrup. Can. J. Chem. 77, 1085 (1999).

30. M. Meyer and J. P. Klinman. Unpublished results.

31. F. H. Westheimer. Chem. Rev. 61, 265 (1961). 